\title{
Spatial Representations in Rat Orbitofrontal Cortex
}

\author{
Andrew M. Wikenheiser, ${ }^{1,2}$ Matthew P. H. Gardner, ${ }^{3}$ Lauren E. Mueller, ${ }^{3}$ and ${ }^{\circledR}$ Geoffrey Schoenbaum ${ }^{3}$ \\ ${ }^{1}$ Department of Psychology, University of California, Los Angeles, Los Angeles, California 90095, ${ }^{2}$ Brain Research Institute, University of California, \\ Los Angeles, Los Angeles, California 90095, and ${ }^{3}$ Behavioral Neurophysiology Research Section, Cellular Neurobiology Research Branch, Intramural \\ Research Program, National Institute on Drug Abuse, Baltimore, Maryland 21224
}

The orbitofrontal cortex (OFC) and hippocampus share striking cognitive and functional similarities. As a result, both structures have been proposed to encode "cognitive maps" that provide useful scaffolds for planning complex behaviors. However, while this function has been exemplified by spatial coding in neurons of hippocampal regions-particularly place and grid cells-spatial representations in the OFC have been investigated far less. Here we sought to address this by recording OFC neurons from male rats engaged in an open-field foraging task like that originally developed to characterize place fields in rodent hippocampal neurons. Single-unit activity was recorded as rats searched for food pellets scattered randomly throughout a large enclosure. In some sessions, particular flavors of food occurred more frequently in particular parts of the enclosure; in others, only a single flavor was used. OFC neurons showed spatially localized firing fields in both conditions, and representations changed between flavored and unflavored foraging periods in a manner reminiscent of remapping in the hippocampus. Compared with hippocampal recordings taken under similar behavioral conditions, OFC spatial representations were less temporally reliable, and there was no significant evidence of grid tuning in OFC neurons. These data confirm that OFC neurons show spatial firing fields in a large, two-dimensional environment in a manner similar to hippocampus. Consistent with the focus of the OFC on biological meaning and goals, spatial coding was weaker than in hippocampus and influenced by outcome identity.

Key words: cognitive map; grid cells; hippocampus; orbitofrontal cortex; place cells; rat

Significance Statement

The orbitofrontal cortex (OFC) and hippocampus have both been proposed to encode "cognitive maps" that provide useful scaffolds for planning complex behaviors. This function is exemplified by place and grid cells identified in hippocampus, the activity of which maps spatial environments. The current study directly demonstrates very similar, though not identical, spatial representatives in OFC neurons, confirming that OFC-like hippocampus-can represent a spatial map under the appropriate experimental conditions.

\section{Introduction}

Convergent work has found striking functional similarities between the orbitofrontal cortex (OFC) and hippocampus (Ramus et al., 2007; Wikenheiser and Schoenbaum, 2016; Guise and Shapiro, 2017). However, it is unclear to what extent spatial coding, a hallmark of hippocampal function (O'Keefe and Nadel, 1978; Redish, 1999; Knierim, 2015), occurs in the OFC. While place cell-like representations have been reported in anterior

Received Apr. 17, 2021; revised June 9, 2021; accepted June 20, 2021.

Author contributions: A.M.W., M.P.H.G., L.E.M., and G.S. designed research; A.M.W. and L.E.M. performed research; A.M.W. analyzed data; G.S. wrote the paper.

The authors declare no competing financial interests.

This work was supported by the Intramural Research Program at the National Institute on Drug Abuse (Grant ZIA-DA000587). The opinions expressed in this article are the authors' own and do not necessarily reflect the view of the National Institutes of Health/Department of Health and Human Services.

Correspondence should be addressed to Geoffrey Schoenbaum at geoffrey.schoenbaum@nih.gov or Andrew M. Wikenheiser at amwikenheiser@psych.ucla.edu.

https://doi.org/10.1523/JNEUROSCI.0830-21.2021

Copyright $\odot 2021$ the authors cingulate, prelimbic, and infralimbic cortices (Hok et al., 2005; Remondes and Wilson, 2013; Powell and Redish, 2014; Mashhoori et al., 2018; Zielinski et al., 2019; Hasz and Redish, 2020; Tang et al., 2021), evidence for similar representations in OFC neurons is weaker (Feierstein et al., 2006; Grieves and Jeffery, 2017; Yang and Masmanidis, 2020).

For example, in operant decision-making tasks, studies frequently identify strong neural correlates of response direction in OFC neurons (Feierstein et al., 2006; Roesch et al., 2006), while such activity may reflect differing spatial information, there is also a confound with past cues and impending reward. Indeed, when response direction and these factors are dissociated in primate studies, directional correlates are reportedly rare (PadoaSchioppa and Assad, 2006; but see Strait et al., 2016). Similarly, OFC recordings on maze-based decision-making tasks find spatial regularities in OFC cell firing; however, these correlates are interpreted as reflecting reward expectation/delivery or associative rather than spatial information (Young and Shapiro, 2011; Steiner and Redish, 2012; Stott and Redish, 2014; Riceberg and 
Shapiro, 2017). Perhaps the clearest demonstration of place as a modulator of OFC activity independent of reward comes from work showing that OFC cell firing discriminated odor ports located on different walls of an operant chamber. Yet, such activity clearly clustered near preferred areas, and work was never done to identify whether such firing was mostly driven by the spatial location of the odor port versus the sensory and associative information (Lipton et al., 1999). Thus, outside the confines of operant boxes and relatively constrained maze-based tasks, tests of OFC representations in two-dimensional spaces are lacking.

The question of spatial representations in OFC has come to the fore recently for two reasons. First, it has been argued that both OFC and hippocampus encode "cognitive maps" that provide useful scaffolds for planning complex behaviors (Cohen and Eichenbaum, 1993; Wilson et al., 2014; Farovik et al., 2015; Wikenheiser and Schoenbaum, 2016; Whittington et al., 2019; Zhou et al., 2019b). This framework invites the obvious question of whether similarities between OFC and hippocampus extend to the level of spatial responses in individual OFC neurons. Second, recent neuroimaging and theoretical work has suggested that spatial coding schemes first identified in the rodent hippocampal system might in fact be general representational mechanisms for organizing information (Behrens et al., 2018). Specifically, signatures of grid-like representations, most often associated with the rodent entorhinal cortex (Hafting et al., 2005; Moser et al., 2008), have been identified in the $\mathrm{OFC}$ and associated regions in human participants trained to navigate abstract relational stimulus spaces (Constantinescu et al., 2016; Bao et al., 2019). However, while these representations show up in BOLD response, it is unclear whether the spiking activity of single neurons in the OFC show grid-like representations.

Here we sought to address these questions by recording OFC neurons from rats engaged in the classic open-field foraging task originally developed to characterize place fields in rodent hippocampal neurons (Muller et al., 1987; Muller and Kubie, 1987). Neural activity was recorded as rats searched for food pellets scattered randomly throughout a large enclosure. Because OFC is known to care about features of appetitive outcomes like flavor, in some sessions, we arranged for particular flavors to occur more frequently in particular parts of the enclosure; in other sessions, only a single flavor was used. Data were analyzed for evidence of spatial firing fields, grid-like representations, and other correlates; comparisons were made to hippocampal recordings made in a similar open-field setting by the Buzsáki group (Mizuseki et al., 2009a,b).

\section{Materials and Methods}

Experimental subjects. Subjects were four experimentally naive, male, Long-Evans rats purchased from Charles River. Rats were $\sim 3$ months of age and weighed $250-275 \mathrm{~g}$ at the beginning of the experiment. Rats were maintained on a $12 \mathrm{~h}$ light/dark cycle and housed singly in a facility accredited by the Association for Assessment and Accreditation of Laboratory Animal Care. During behavioral testing and recording sessions, rats were food restricted; rats were weighed daily and provided supplemental food in addition to what they earned on the behavioral task to maintain their weight at $>85 \%$ of their free-feeding weight. Rats were handled for $5 \mathrm{~d}$ before behavioral training commenced.

Behavioral apparatus. Rats were trained to search a square, openfield arena for food pellets. The arena was $122 \times 122 \mathrm{~cm}$, and enclosed on all four sides by opaque $50 \mathrm{~cm}$ walls. The arena was surrounded by black curtains, and a white cue card was affixed on one of the walls to provide a salient visual cue to orient the rats. Four food-pellet dispensers
(Colbourn) were mounted above the arena, and connected via plastic tubing to custom, 3D-printed attachments positioned $\sim 60 \mathrm{~cm}$ above the arena, at the center of each of the four walls. These attachments controlled the angle at which dispensed pellets dropped into the arena and were designed to control the distribution of food pellets from each feeder and compose the flavor zones depicted in Figure $1 a$. Figure $1 a$ was constructed by firing 100 pellets from each feeder and manually counting where each pellet came to rest in a grid superimposed over the arena. One type of $20 \mathrm{mg}$ food pellet was delivered from each feeder to define the standard flavor zones, which were identical for all rats. The four food pellet flavors used for the standard flavor zones were fruit punch, raspberry, peanut butter, and banana.

Behavioral training and testing. Rats were habituated to the openfield arena by allowing them to explore it for 5-10 min on several consecutive days, after which behavioral training began. During training sessions, rats were placed in the center of the arena and food pellets were dispensed at random intervals. Interpellet intervals were drawn from a Gaussian distribution with a mean of $30 \mathrm{~s}$ and a variance to $10 \mathrm{~s}$ (rare negative values were removed). The identity of which feeder was activated was pseudorandomly determined such that for every 12 food pellet deliveries, each of the four feeders was activated three times, with the order of activation randomized. Pellet delivery was in no way contingent on the behavior of the rats. Daily training sessions lasted for $30 \mathrm{~min}$ and continued for 3 weeks to ensure that rats had sufficient opportunity to learn the flavor-location associations instantiated by the task.

Neural recordings. Rats were implanted with custom electrode arrays targeting OFC bilaterally, following procedures that have been detailed previously (Roesch et al., 2006; Stalnaker et al., 2014; Wikenheiser and Schoenbaum, 2016). For three rats, recording probes consisted of two drivable bundles of 16 formvar-insulated nichrome wires (diameter, $25 \mu \mathrm{m}$; A-M Systems). For one rat, recording probes consisted of two drivable bundles of eight stereotrodes, constructed by twisting together strands of 17.78- $\mu \mathrm{m}$-diameter nichrome wire (A-M Systems). All electrodes were electroplated to an impedance of $\sim 200 \mathrm{k} \Omega$ measured at $1000 \mathrm{~Hz}$ using gold plating solution (Neuralynx). Electrode bundles were implanted $3.0 \mathrm{~mm}$ anterior to bregma and $\pm 3.3 \mathrm{~mm}$ lateral to bregma. During surgery, electrode bundles were lowered $4 \mathrm{~mm}$ ventral to brain surface and secured via dental cement. Rats recovered for 5-7 d after surgery, during which time electrodes were advanced to reach dorsal OFC. After each recording session, electrodes were advanced $40-80 \mu \mathrm{m}$ to sample new neurons. Recording sites were reconstructed histologically and confirmed to lie with the lateral orbitofrontal and ventral anterior insular regions.

Neural recordings were collected using an OpenEphys acquisition system. Electrode signals were amplified and digitized by a 32-channel Intan headstage connected to the neural recording probe. Each channel was sampled continuously at $30 \mathrm{kHz}$, referenced to a common average signal that included all non-noisy channels, and filtered between 600 and $6000 \mathrm{~Hz}$. Spike thresholds were set manually before each recording session. Threshold-crossing events were sorted into putative single units post hoc using MClust (http://redishlab.neuroscience.umn.edu/MClust/ MClust.html), following standard spike-sorting procedures.

As a comparison to our OFC data, we also downloaded and analyzed an online dataset of hippocampal recordings (Mizuseki et al., 2009a,b). These data have been described in detail previously. Briefly, rats foraged for drops of water for $\sim 50 \mathrm{~min}$ in a $120 \mathrm{~cm}$ square-shaped open field similar to the one used in our experiment. Recordings were made in right dorsal CA1 hippocampus using 64-channel eight-shank silicon probes.

Session sequence. After recovery from surgery, rats first performed $30 \mathrm{~min}$ sessions of the flavor gradient foraging task. These were identical to presurgery behavioral training sessions. When performance returned to presurgery levels for at least four consecutive sessions, rats were tested on a sequence of two-part recording sessions. In flavored-unflavored sessions, flavored pellets were delivered in their usual locations for one portion of the session, and a uniform distribution of unflavored, $20 \mathrm{mg}$ sucrose pellets was delivered in a second portion of the session. The flavored and unflavored foraging periods each lasted $20 \mathrm{~min}$, and were separated by $\sim 10 \mathrm{~min}$, during which the rat rested outside the enclosure. 
a
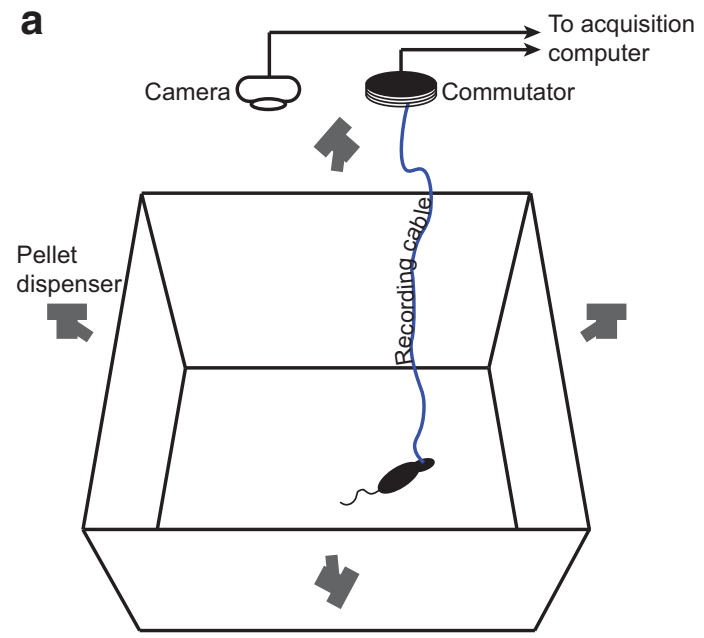

b

Flavor zones
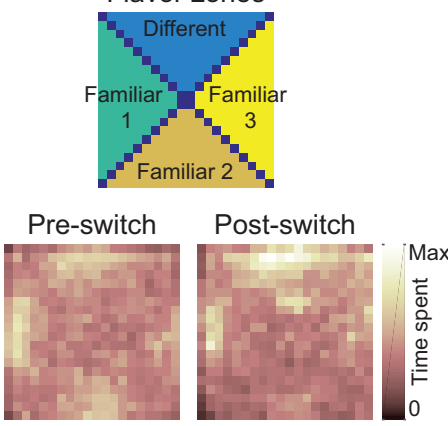

d
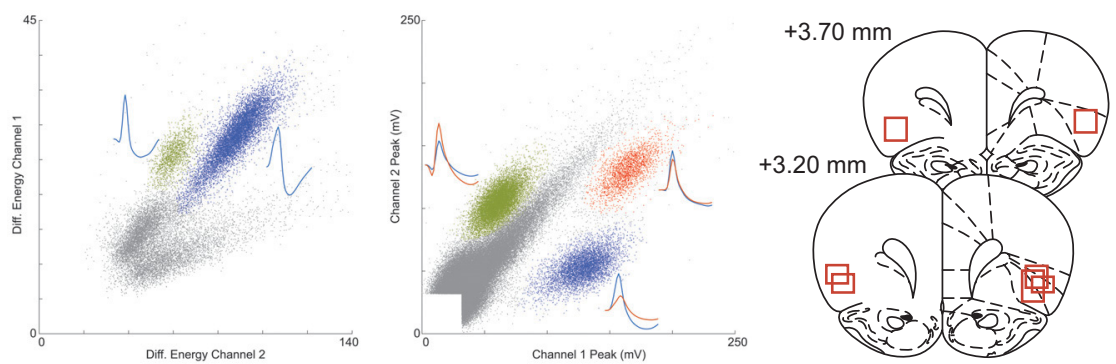

Figure 1. Behavioral task and probe test behavior. $\boldsymbol{a}$, Rats were tested in a large, open-field endosure surrounded by food pellet dispensers (left). Four flavors of sucrose pellets were delivered in four spatial flavor zones (right), such that each of the four flavors was most likely to be found in a particular location (top), but the probability of finding any pellet was even across the enclosure. Color indicates the probability of finding pellets of individual pellets (top) and any pellet (bottom). Data were generated by delivering 20 pellets of each flavor and counting the number that landed at each location within the arena. $\boldsymbol{b}$, In probe sessions, rats spent similar amounts of time in each flavor zone for the first foraging period, when flavors were delivered in their familiar locations. However, when one familiar flavor was switched to a novel pellet type, rats distributed more time to searching the pellet zone corresponding to the novel flavor. Color indicates the grand average of time spent at each location, across four probe sessions for each of four rats. c, Quantification of data in b. Mean time in each flavor zone was obtained by averaging pixels defining each zone, as shown in the far left panel of $\boldsymbol{b}$. $\boldsymbol{d}$, We recorded neurons bilaterally in OFC using single wire electrodes (left; $n=3$ rats; 16 electrodes per hemisphere) or stereotrodes (right; $n=1 ; 8$ stereotrodes per hemisphere). $\boldsymbol{e}$ Histologic reconstruction of recording sites confirmed that electrode placements were localized to the lateral orbitofrontal and ventral anterior insular regions. Max, Maximum; Diff., different.

Each rat performed a total of eight flavored-unflavored sessions, with the flavored period occurring in the first period for half of sessions and in the second period for the remainder. Intermixed with flavored-unflavored sessions, rats also performed two flavored-flavored sessions, which were structurally identical to flavored-unflavored sessions, except that flavored pellets were delivered in their previously established locations for both portions of the session. The order of the eight flavored-unflavored sessions and two flavored-flavored sessions was randomized for each rat. Data from three flavored-unflavored sessions were lost because of a hardware synchronization issue. Finally, after the completion of neural recordings, rats underwent a series of four probe sessions. In probe sessions, rats foraged under the standard flavor gradient conditions for $20 \mathrm{~min}$, and under a modified version of the flavor gradients for a second 20 min period; in this second period, flavor gradients were modified by replacing one of the usual pellet flavors with a novel type that rats had never encountered on the task arena previously, but which they had consumed previously in their home cage (substitute pellet flavors: grape, grain, chocolate, and bacon). Rats underwent four probe sessions, such that each of the four familiar flavors were altered in the second half of one probe session. Table 1 summarizes the sessions each rat completed and the number of neurons recorded under each condition.

Firing rate maps. Firing rate maps were constructed by counting the number of spikes a cell fired while the rat was in each of $30 \times 30, \sim 4 \mathrm{~cm}^{2}$ bins, and dividing by the total amount of time rats spent in each bin. Periods of slow movement (running speed, $<5 \mathrm{~cm} / \mathrm{s}$ ) were excluded. Firing rate was not estimated for bins with a total visit duration of $<0.2 \mathrm{~s}$. For display only, firing rate maps were smoothed by convolution with a $2-\mathrm{D}$ Gaussian kernel with $\sigma$ of 1 bin; all analyses involving rate maps were performed on unsmoothed rate maps.

Spatial information. Using methods developed by others (Skaggs et al., 1992; Markus et al., 1994; Diehl et al., 2017), spatial information per spike was computed for each firing rate map using the following equation: $I=\sum_{i} P_{i}\left(R_{i} / R\right) \log \left(R_{i} / R\right)$, where $I$ indexes the spatial bins in the rate map, $P_{i}$ is the occupancy probability of each bin, $R_{i}$ is the firing rate in each bin, and $R$ is the average firing rate across bins. To test for significant spatial representations, the spike train of each cell was shifted relative to videotracking data by a fixed random offset drawn from a uniform distribution ranging from 10 to $30 \mathrm{~s}$. Using the shifted spike train, a rate map and spatial information score were recomputed; this process was repeated 1000 times for each cell. Cells with a peak spatial firing rate $>2 \mathrm{~Hz}$ and a spatial information score $>95 \%$ of shuffled rate maps were counted as having significant spatial representations.

Firing field center locations. To examine the distribution of firing fields across the arena (see Figs. 3, 4), firing fields were defined as contiguous pixels with a mean firing rate $\geq 40 \%$ of the maximum firing rate of the cell. In addition, individual fields whose area was $<1 \%$ of the enclosure or $>25 \%$ of the enclosure were excluded from the field center analysis. Note that such cells might still encode spatial information; however, for cells whose firing is distributed widely over space, the concept of the center of the field becomes less meaningful, so such neurons ( 66 of 487 cells, $\sim 13.5 \%$ ) were excluded from this analysis. More than one field could be detected per cell; in this case, the field with the highest average firing rate was used in constructing plots of field centers.

Spatial cross-correlation. For spatial cross-correlation analyses (see Fig. 5), rate maps for each cell were computed separately for the different 
Table 1. Number of sessions and cells recorded by session type

\begin{tabular}{lcccc}
\hline Rat & Nonswitch & $\begin{array}{l}\text { Switch } \\
\text { (Flav } \rightarrow \text { Unflav) }\end{array}$ & $\begin{array}{l}\text { Switch } \\
\text { (Flav } \rightarrow \text { Flav) }\end{array}$ & Probe \\
\hline Number of sessions by session type & & & & \\
1 & 4 & 7 & 2 & 4 \\
2 & 4 & 7 & 2 & 4 \\
3 & 4 & 7 & 2 & 4 \\
4 & 4 & 8 & 8 & 16 \\
Total & 16 & 29 & & \\
Cells recorded by session type & & & 23 & \\
1 & 41 & 65 & 40 & \\
2 & 39 & 80 & 23 & \\
3 & 33 & 64 & 19 & \\
4 & 72 & 93 & 105 & \\
Total & 185 & 302 & & \\
\hline
\end{tabular}

Flav, Flavored; Unflav, unflavored.

conditions under investigation (i.e., flavor zone foraging period vs foraging for a uniform distribution of unflavored pellets). Cross-correlograms were computed by shifting one rate map relative to the other in the $x$ and $y$ directions and taking the pixel-by-pixel correlation where the two rate maps overlapped. A shift of zero for both $x$ and $y$ corresponds to a perfect alignment of rate maps; therefore, two rate maps with similar spatial firing patterns should produce high correlation values for small shifts, more distinct representations across conditions result in low correlation values. To further visualize how cross-correlation changed with increasingly large shifts (see Fig. 5b), we averaged cross-correlograms over all cells in flavored-flavored and flavored-unflavored sessions (see Fig. $5 a$ ), and then plotted the value of each pixel in the average cross-correlogram as a function of the distance to the origin of that pixel.

Cross-temporal correlation. We performed a further correlation analysis to measure representation stability over time, both with and across flavor contexts. For each cell recorded in flavored-flavored and flavoredunflavored sessions, rate maps were computed in 6 min sliding windows, which were stepped across each session half in $3.5 \mathrm{~min}$ steps. Thus, the first rate map spanned 0-6 $\mathrm{min}$, the second spanned 3.5-9.5 $\mathrm{min}$, the third spanned 7-13 min, and so on. For each cell, this procedure resulted in 5 rate maps for each session half, or 10 rate maps for each session. Next, the correlation between every pair of rate maps of a cell was taken to produce the correlation matrices seen in Figure 6. As before, the spatial firing rate was not estimated for bins that the rat visited for $<0.2 \mathrm{~s}$, so these locations were not included in computing correlations. Correlation matrices were averaged across all neurons recorded in a particular session type to construct the average plots seen in Figure $6 a$.

Gridness analysis. To test for grid-like representations, we followed the approach outlined by Brandon et al. (2011) for computing the expanding gridness score with elliptical correction for each OFC neuron recorded in nonswitch or flavored-unflavored task sessions. Briefly, spatial autocorrelograms were constructed for each cell following the same approach described for the spatial cross-correlation analysis, but shifting a copy of the rate map relative to itself and computing the pixel-by-pixel correlation of overlapping portions. The correlation peak at the center was removed by defining an inner radius with a length of half the average distance to the four correlogram peaks nearest to the origin. Next, an annulus was extracted by choosing an outer radius with length greater than the inner radius. The annulus was then rotated relative to itself, the correlation was computed for every rotation, and plots of correlation as a function of rotation (see Fig. $7 b$ ) were examined for periodicity consistent with grid-like representations. The gridness score was defined as the difference between the minimum correlation value at $60^{\circ}$ or $120^{\circ}$ rotations and the maximum correlation value at $30^{\circ}, 50^{\circ}$, and $90^{\circ}$ rotations. To mitigate potential difficulties in correctly choosing the length of the outer radius, we tested all possible lengths and report the greatest resulting gridness score. Because standard measures of gridness are sensitive to minor elliptical distortions in the arrangement of grid fields, we computed two possible corrections by defining the length of the long and short axes of the ellipse as the distance from the origin to the farthest and nearest autocorrelogram peaks, respectively. For each correction, the length of the missing elliptical axis was inferred as described previously (Brandon et al., 2011), and the autocorrelogram was warped to equalize the lengths of these axes. Gridness scores were computed for both corrections using the expanding outer radius approach outlined above, and the higher of the two scores is reported.

To test for significant grid tuning, we shifted spikes relative to videotracking data as described above and recomputed the gridness score for each shifted spike train. This process was repeated 500 times to generate a null distribution of gridness scores, and cells whose gridness score exceeded $95 \%$ of shuffled scores were considered to have significant grid tuning. This significance testing procedure has been shown to have a higher than expected false-positive rate in simulated data with irregularly distributed spatial firing fields (Barry and Burgess, 2017). Thus, though few OFC cells reached significance using the approach outlined above, it is possible that with a more stringent "field shuffling" test of significance even fewer cells would have been identified as having grid-like tuning.

Analyses of hippocampal recordings. The hc- 2 neural recording dataset was downloaded from CRCNS.org (Mizuseki et al., 2009a) and was analyzed to provide a comparison between hippocampal and OFC neurons recorded under similar circumstances. We analyzed 148 neurons from four sessions recorded under these conditions. All analyses of hippocampal units were performed following the same approaches as detailed above for OFC neurons.

\section{Results}

We recorded neurons in the lateral OFC as rats performed a foraging task inspired by the behavioral paradigms used in classic work investigating spatial representations (Fig. 1a). Rats were placed in a large $(122 \times 122 \mathrm{~cm})$, square-shaped enclosure, where flavored sucrose pellets were delivered from four dispensers mounted above the enclosure with an average frequency of two pellets per minute. The random scatter of pellets encouraged rats to fully explore the enclosure, allowing us to assess the firing rates of neurons at each location in the enclosure. The task also ensured that behavioral patterns associated with seeking or consuming food pellets were distributed randomly over space, so that neural responses specific to such behaviors could not be mistaken for spatial correlates.

Our version of the task was modified to provide a richer appetitive outcome structure aimed at better engaging the OFC. Four different flavors of nutritionally equivalent and similarly valued sucrose pellets were delivered within separate spatial zones across the arena (Fig. 1b, top). We did not conduct preference tests for pellet types outside of the foraging task context, but behavioral data from probe test sessions (described below) suggests that the rats did not have strong preferences among the four flavors. The likelihood of finding a pellet (regardless of its flavor) was uniform across the arena (Fig. $1 b$, bottom), but each of the four flavors was most likely to be encountered within its flavor zone. Thus, the expected pellet flavor varied over space, while the average density of pellets did not.

\section{Rats responded to violations of flavor structure}

To test whether rats attended to the similarly valued flavors being delivered in the different zones, probe sessions were conducted after the collection of neural data were complete. Probe sessions were divided into two 20 min foraging periods. During the first portion of probe sessions, rats searched for pellets delivered in the four flavor zones, as described above. During the second portion of the probe session, one flavor zone was altered by switching the usual pellet for that zone with a flavor that the rat had not encountered before on the task (but which had been experienced previously in the home cage to habituate neophobia). The 

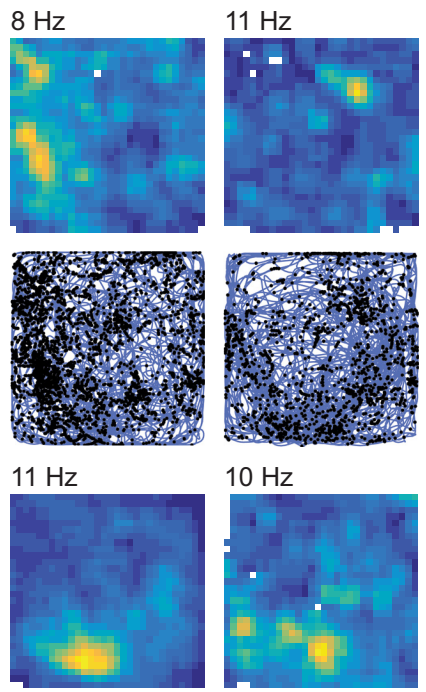

$10 \mathrm{~Hz}$
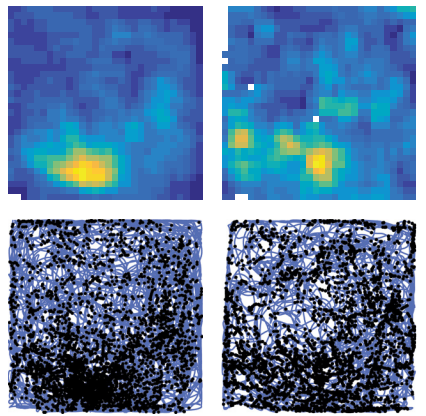

0
$8 \mathrm{~Hz}$
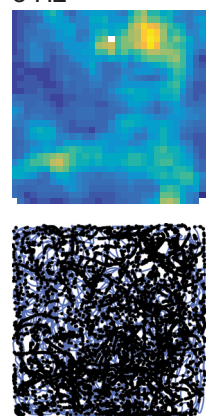

$10 \mathrm{~Hz}$
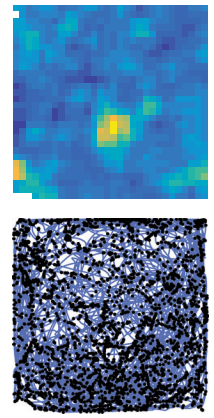

$15 \mathrm{~Hz}$
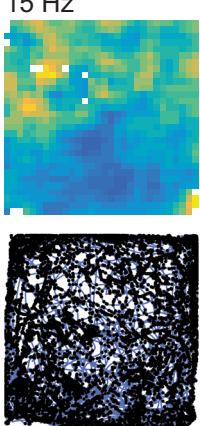

$15 \mathrm{~Hz}$
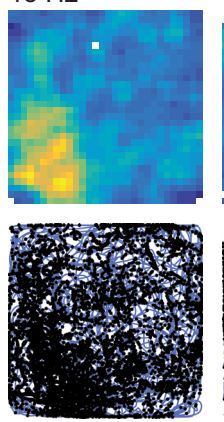

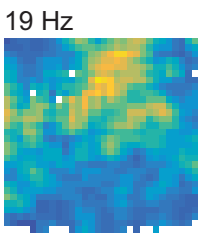

$22 \mathrm{~Hz}$
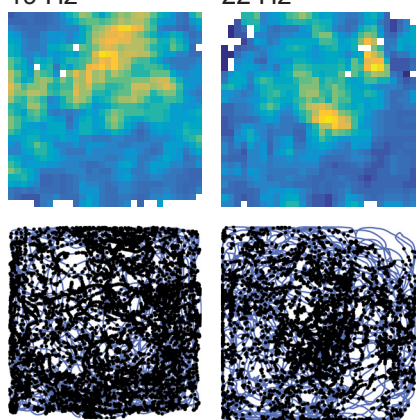

$12 \mathrm{~Hz}$
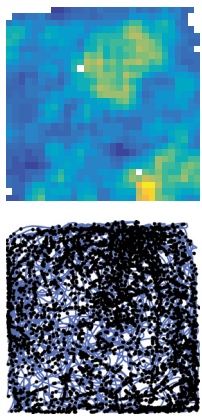

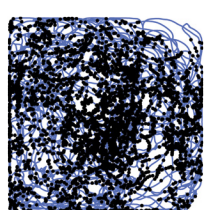

$8 \mathrm{~Hz}$
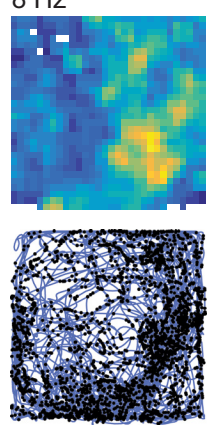

Figure 2. Example cells. Examples of spatial representations in 12 OFC neurons are shown. Heat maps show firing rate maps constructed by dividing the enclosure into a $30 \times 30$ grid of bins and computing the session average firing rate for each location. The maximum firing rate of each cell is plotted near the upper left corner of firing rate maps. Regions of the enclosure that rats did not spend at least $0.2 \mathrm{~s}$ in over the course of the 30 min session are represented with a white pixel. Below each firing rate map, a corresponding plot of the location of the rat (blue) and the location of each action potential (black dots) is shown. Max, Maximum.

order in which the flavor zones were altered during probe sessions was randomized, and across four probe sessions all four flavor zones were substituted for each rat.

We divided the enclosure into four flavor zones defined by the pellet flavor probability gradients (Fig. 1c, top) and measured how much time rats spent searching in each location before and after the introduction of the different pellet type. In the first half of probe sessions, rats spent approximately equal amounts of time in the four flavor zones; however, when a new flavor was introduced, the rats spent significantly more time in the zone containing that pellet type (Fig. $1 c, d$, Post-switch). A two-way ANOVA on time spent in each zone showed a main effect of flavor zone $\left(F_{(3,120)}=10.92, p=2.14 \times 10^{-6}\right)$ and a zone $\times$ session half (familiar flavor vs different flavor portion of the session) interaction $\left(F_{(3,120)}=5.89, p=8.69 \times 10^{-4}\right)$. The main effect of session half was not significant $\left(F_{(1,120)}=0.23, p=0.63\right)$. Bonferroni-corrected post hoc paired $t$ tests showed that these significant effects reflected a significant increase in time spent in the different flavor zone during the second half of probe sessions relative to the first half of the session when the familiar pellet type was delivered $\left(t_{(15)}=-6.72, p=2.73 \times 10^{-6}\right)$. Familiar zones one and two showed concomitant decreases in search time during the second half of probe sessions (familiar zone one: $t_{(15)}=-3.93$, $p=5.30 \times 10^{-3}$; familiar zone two: $t_{(15)}=5.65, p=1.85 \times 10^{-4}$ ), while there were no significant change for familiar zone three $\left(t_{(15)}=-1.51, p=0.59\right)$. These data suggest that rats generally valued the familiar pellets similarly; however, they were attending to the different flavors and recognized alterations to the flavor structure of the task, directing their search efforts toward regions where violations of expectations occurred (Costa and Averbeck, 2020).

\section{OFC neurons showed spatial representations}

We recorded lateral OFC neurons as rats performed the flavor zone task (Fig. 1e,f). A complete summary of the number of cells recorded from each rat is given in Table 1. To look for evidence of spatial representations, we divided the enclosure into a $30 \times 30$ grid of $\sim 4 \mathrm{~cm}^{2}$ bins and computed the average firing rate of each neuron for every location. Locations that rats did not visit for at least $0.2 \mathrm{~s}$ are represented by white pixels in firing rate maps. Many OFC neurons showed distinct spatial firing fields (Fig. 2). We computed the spatial information score (Skaggs et al., 1992; Markus et al., 1994; Diehl et al., 2017) for each firing rate map. We also shifted each spike train relative to the tracking data of the position of the rat by a random offset, and recomputed firing rate maps and spatial information scores; this process was repeated 500 times, and neurons were considered to have significant spatial representations if their spatial information score exceeded that of $95 \%$ of the shifted spike trains. By this metric, 64 of 185 OFC neurons recorded on the flavor zone task (34.5\%) showed significant spatial tuning (Fig. $3 a$ ). Spatially tuned neurons typically showed firing fields that remained stable for the duration of the recording session (Fig. $3 b-e$ ). The firing fields of spatially tuned neurons were evenly distributed across the four flavor zones (Fig. $3 f ; \chi^{2}=2.16$; $\mathrm{df}=3, N=50, p=0.20$ ).

\section{Spatial tuning was not driven by flavor selectivity, but was sensitive to flavor context}

Previous work has established that OFC neurons often encode information about the properties of appetitive outcomes, such as flavor. Thus, OFC neurons that responded to consumption of a particular pellet flavor (or encoded memories or expectations of such experiences) could manifest a crude spatial representation, confined to the flavor zones over which each pellet type was 
delivered. The firing fields of OFC neurons were not constrained by the flavor zones we imposed in this way (Fig. 2). However, the flavor structure of the environment may still have modulated firing patterns in OFC neurons. In hippocampal neurons, for instance, changing features of salient sensory cues in the environment can induce "remapping," causing neurons to gain or lose place fields, or form place fields in new locations.

To test for such effects directly, we recorded OFC neurons in a version of the task where location was correlated with or independent of flavor at different times in individual sessions. In these flavored-unflavored sessions, rats foraged for two, $20 \mathrm{~min}$ periods separated by 10 min of rest outside the enclosure. For one foraging period, flavored pellets were delivered in their familiar flavor zones as before; for the other foraging period, unflavored but also familiar sucrose pellets were delivered uniformly over the enclosure. The order of flavored and unflavored foraging periods was randomized and counterbalanced across sessions.

Of 302 neurons recorded during flavored-unflavored sessions, 73 neurons showed significant spatial tuning during the flavored foraging period, while 100 neurons showed significant spatial tuning during the unflavored portion (Fig. 4a). The mean spatial information score of significant neurons did not differ between flavored and unflavored periods of the session $\left(\mu_{\text {flavored }}=\right.$ $0.96 \mathrm{bits} / \mathrm{s} ; \mu_{\text {unflavored }}=0.82 \mathrm{bit} / \mathrm{s} ; p=0.30 ; t_{(171)}=1.03$; two-sample $t$ test), and the firing fields of spatially tuned neurons were evenly distributed across the four flavor zones, both during flavored and unflavored portions of sessions (Fig. $4 b$; flavored portion: $\chi^{2}=1.87$; $\mathrm{df}=3, N=53, p=0.21$; unflavored portion: $\chi^{2}=$ 4.10; $\mathrm{df}=3, N=63, p=0.10)$. These data indicate that similar numbers of OFC neurons showed spatial tuning whether or not flavors were arranged in spatial patterns in the environment.

Yet, while spatial tuning was present in both portions of the task, relatively few neurons retained significant spatial tuning for both flavored and unflavored portions of the task, suggesting that flavor context may modulate OFC spatial representations. Indeed, of the 149 OFC neurons that showed spatial tuning for at least one foraging period of flavored-unflavored sessions, only $16.1 \%$ (24 neurons) had significant spatial tuning for both the flavored and unflavored portions (Fig. $4 c$, left). To explore the tendency of neurons to gain or lose tuning between the flavored and unflavored portion of these sessions, we also recorded neurons on flavored-flavored sessions. These sessions were structurally identical to flavored-unflavored sessions except that both 20 min foraging periods were in the flavor zone context. Of 105 OFC neurons recorded in flavored-flavored sessions, 37 neurons showed significant spatial representations in the first flavored period, while 38 neurons showed significant spatial tuning in the second flavored period. Of the neurons with significant spatial tuning in at least one period of the session, $36.3 \%$ were significant for both periods (Fig. $4 c$, right). Notably, the proportion of neurons that maintained spatial tuning in both session periods was significantly greater for flavored-flavored sessions $\left(z_{(42)}=-\right.$ $3.12 ; p=1.79 \times 10^{-3} ; z$-test for population proportions), suggesting that spatial tuning in OFC neurons was sensitive to the current flavor context under which animals were foraging.

\section{Spatial representations were more similar within flavor context than across flavor context}

Identifying neurons as spatially tuned does not speak to their actual firing patterns. A neuron may have significant spatial representations in both foraging portions of the task without firing in the same location for both contexts. Hippocampal place cells,
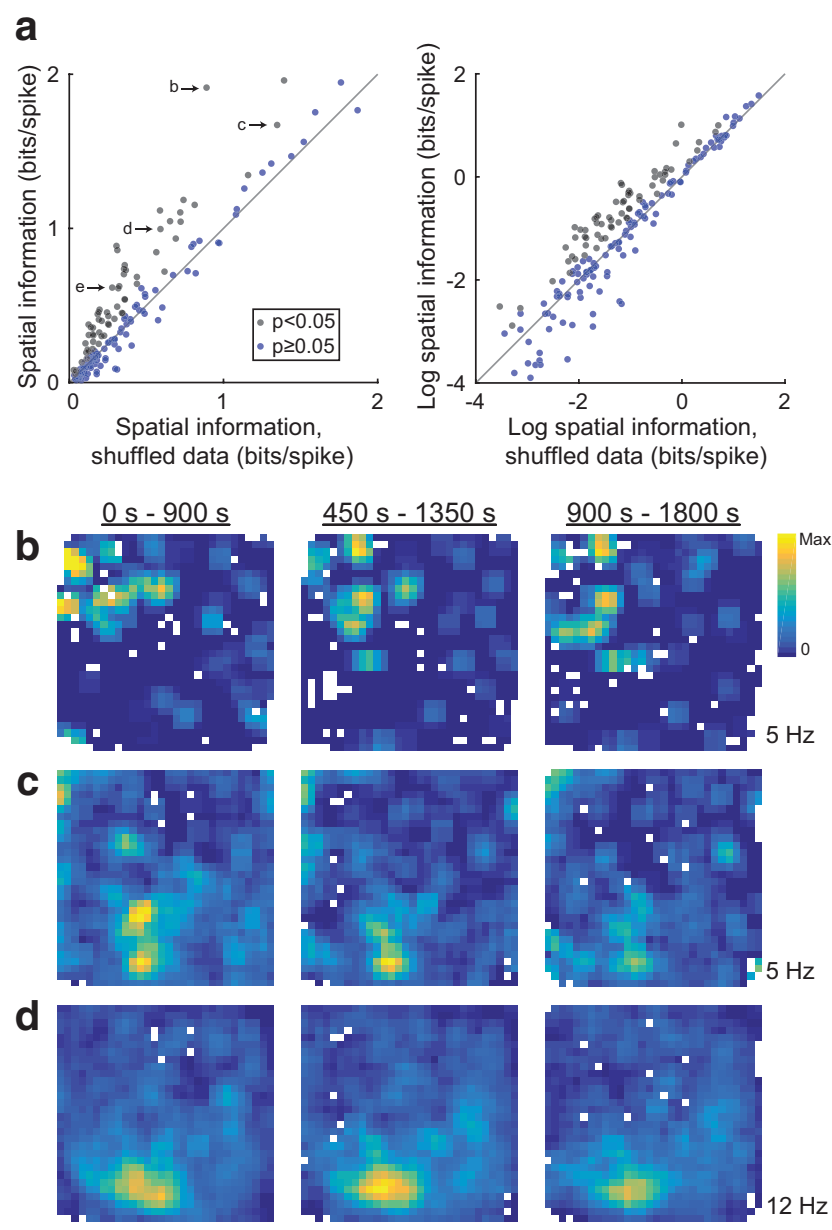

e
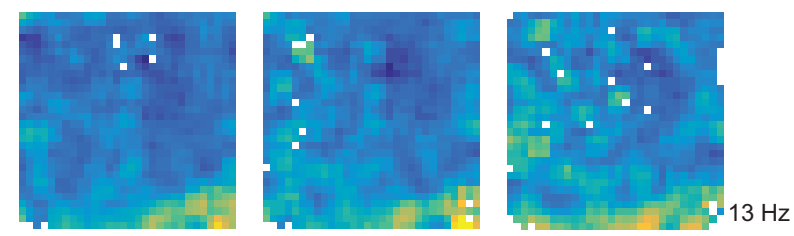

f Field center locations

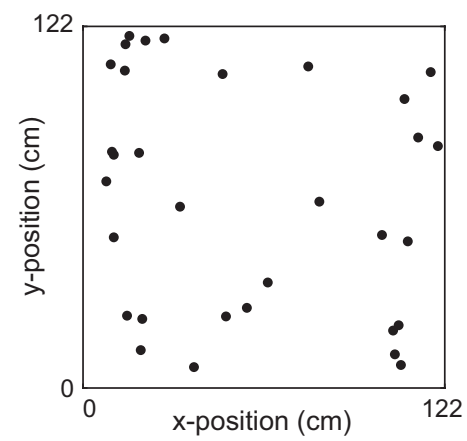

Figure 3. Spatial information in OFC cells. $\boldsymbol{a}$, The spatial information score for each OFC neuron is plotted against the mean spatial information score averaged over 500 random misalignments of the activity of each cell and the location of the rat. Neurons plotted with black dots met the statistical criterion for significant spatial information. $\boldsymbol{b}$, Same as $\boldsymbol{a}$, except the logarithm of spatial information is plotted for better visibility. $\mathbf{c}-\boldsymbol{e}$, Firing rate maps for the neurons labeled in $\boldsymbol{a}$ are computed over three different periods of the recording session. Generally, similar patterns of firing are evident for these neurons throughout the three portions of the recording session. $f$, To examine the distribution of firing fields, we extracted contiguous regions of elevated firing rate, and found the center of such fields. For cells with more than one region of elevated firing, we extracted the field with the greatest average firing rate. Firing fields were distributed relatively uniformly over the enclosure for nonswitch sessions. 
a

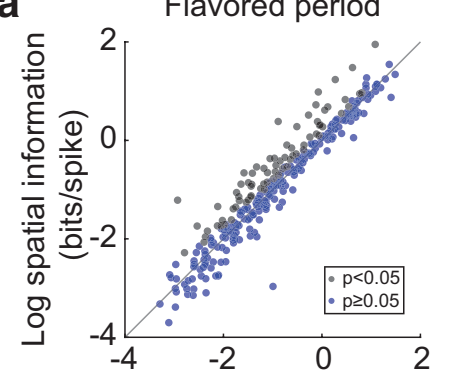

Log spatial information, shuffled data (bits/spike)

b

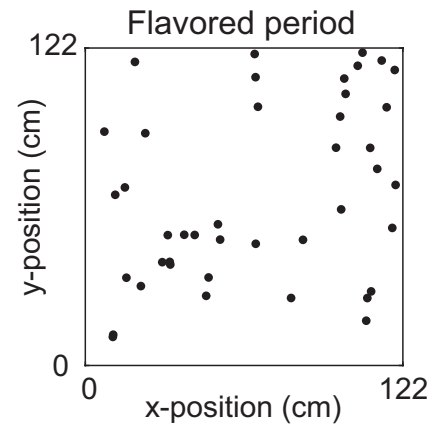

C

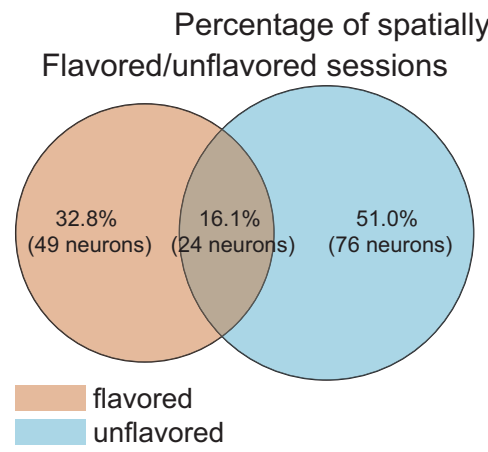

Unflavored period

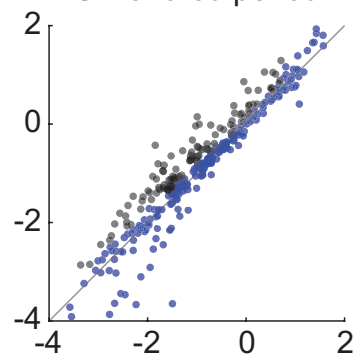

Log spatial information, shuffled data (bits/spike)

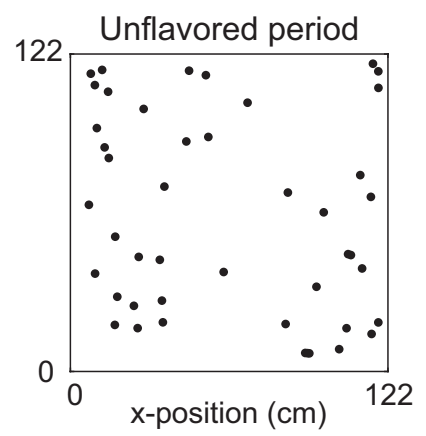

-tuned OFC neurons

Flavored/flavored sessions

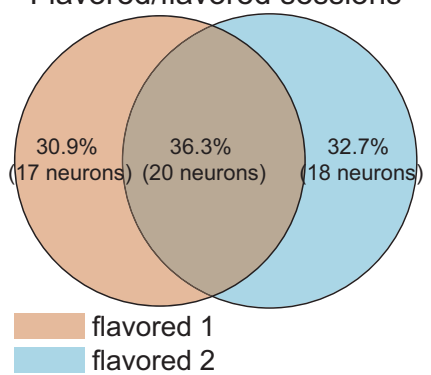

Figure 4. Spatial tuning across contexts. $\boldsymbol{a}$, Many OFC neurons showed significant spatial tuning for both foraging periods of flavored-unflavored sessions. $\boldsymbol{b}$, Firing fields were detected and the locations of their centers were plotted for flavored and unflavored foraging periods. Fields were evenly distributed over the enclosure for both flavored and unflavored foraging periods. $c$, The proportion of neurons with significant spatial tuning during both periods of the session was greater in flavored-flavored sessions than in flavored-unflavored sessions.

for instance, show statistically independent spatial representations across different contexts (Muller and Kubie, 1987; Bostock et al., 1991; Colgin et al., 2008; Kubie et al., 2020).

To investigate this question, we calculated the spatial crosscorrelation between firing rate maps computed separately for each period of flavored-unflavored and flavored-flavored sessions. In this analysis, the pixel-by-pixel correlation is measured between the two firing rate maps as one map is shifted relative to the other. If representations are similar between two firing rate maps, the correlation will be strongest near shifts of zero, corresponding to the origin of 2-D spatial cross-correlograms, and will progressively weaken with increasingly large shifts. Importantly, this method measures the similarity of firing rate maps without making assumptions about the shape or complexity of firing fields.

Both exemplar (Fig. 5a, left column) and average (Fig. 5b, left) spatial cross-correlograms of OFC neurons recorded during flavored-flavored sessions $(N=105)$ showed a high correlation centered around shifts of zero, indicating that representations were-on average-similar between the first and second flavor zone foraging periods of these sessions. By contrast, exemplar (Fig. $5 a$, right column) and average (Fig. 5b, right) spatial cross-correlograms for OFC neurons recorded during flavored-unflavored sessions $(N=305)$ showed a substantially lower correlation around shifts of zero, indicating less similar representations between contexts in these sessions. A statistical analysis showed that the average cross-correlation strength for spatial shifts $<20 \mathrm{~cm}$ was significantly greater for cells recorded in flavored-flavored sessions than it was for cells recorded in flavoredunflavored conditions (Fig. $5 c$; $t_{(405)}=-5.57$; $p=4.60 \times 10^{-8}$; two-sample $t$ test). As described previously, the spatial information of neurons that had firing fields in flavored foraging periods was not significantly different from neurons with firing fields in unflavored sessions, and neither was the proportion of neurons with significant spatial tuning different between flavored and unflavored foraging periods. Thus, at the population level, the quality of OFC spatial representations did not differ between flavored and unflavored contexts. However, the pattern of spatial firing for any individual OFC unit was more likely to change between flavored and unflavored foraging periods than when flavor context was held constant.

We performed an additional correlation analysis to assess how similar representations were across time, both within and across the two session periods. Firing rate maps were computed in sliding windows (window length $=6 \mathrm{~min}$; step size $=3.5 \mathrm{~min}$ ), and the correlation between firing rate maps for each pair of windows was calculated for individual exemplars (Fig. 6a) and averaged across all cells (Fig. 6b). As expected, pixels near the diagonal of the correlation matrix showed strong correlations, indicating that firing rate maps from nearby time bins were more similar than rate maps separated by long durations. However, consistent with our previous analyses, the tuning of neurons recorded in flavored-unflavored sessions showed weaker correlations across the two periods compared with neurons recorded in flavored-flavored sessions. We computed the mean across-context correlation for each neuron by averaging pixels in the lowerleft and upper-right quadrants of the correlation matrix of each cell. The mean within-context correlation was computed analogously by averaging correlation values in the upper-left and lower-right quadrants (excluding pixels along the diagonal where entries always take the maximal value of 1 ). While the strength of the within-context correlation did not significantly differ for neurons recorded in different types of sessions (Fig. $6 c ; t_{(405)}=0.47 ; p=0.64$; two-sample $t$ test), the average between-context correlation was significantly lower for neurons recorded in flavored-unflavored sessions (Fig. $6 c$; $t_{(405)}=4.95 ; p=1.07 \times 10^{-6}$; two-sample $t$ test). These analyses 
indicate that, independent of temporal effects, the spatial tuning in OFC was significantly more stable when flavor remained the same across two contexts than when flavor changed.

\section{Lack of grid-like representations in OFC neurons}

Recent neuroimaging experiments have suggested that grid-like tuning, a spatial firing motif first identified in the entorhinal cortices of rats, may be a general coding mechanism for structuring representations of abstract, nonspatial concepts into a mental map (Hafting et al., 2005; Moser et al., 2008). Organization of representations in this manner could facilitate planning and decision-making, and signatures of such grid-like representations have been identified in portions of frontal cortex, including the OFC (Constantinescu et al., 2016; Bao et al., 2019). It is unknown, however, whether single cells in OFC show grid cell tuning. This is an important question, since BOLD signaling is significantly influenced by afferent input and local processing (Logothetis and Wandell, 2004), whereas fine-wire recording electrodes like those used here are biased to pick up activity from large "regular spiking" putative pyramidal neurons (McCormick et al., 1985; Connors and Gutnick, 1990).

To test for the presence of grid tuning in OFC neurons, we calculated rate maps (Fig. $7 b$, left column), rate map autocorrelograms (Fig. $7 b$, middle column), and gridness scores for each OFC neuron we recorded in the flavored-flavored and flavored-unflavored sessions described above. The gridness score quantifies the sixfold rotational symmetry that is characteristic of grid cells (Sargolini et al., 2006; Brandon et al., 2011; Barry and Burgess, 2017). The correlation is computed between the rate map of each cell and a rotated version of the rate map, with grid tuning resulting in higher correlations at rotations of $60^{\circ}$ and $120^{\circ}$ (Fig. $7 b$, right column). Cells with gridness scores exceeding 95\% of scores generated from randomly shuffled data were considered to have significant grid tuning. Because we found that representations depended on flavor context in our task, we computed grid scores separately for flavored and unflavored portions of flavored-unflavored sessions.

This analysis revealed that grid-like representations were rare in OFC neurons. In nonswitch sessions, only $2 \%$ of cells (4 of 185 cells) showed significant grid tuning, which was not significantly different from the $5 \%$ of neurons expected to pass the statistical threshold for grid-like tuning $(p=0.09$, two-tailed binomial test). Similarly in flavored-unflavored sessions $4 \%$ of cells ( 12 of 302 cells; $p=0.51$, two-tailed binomial test) passed the statistical threshold for grid tuning
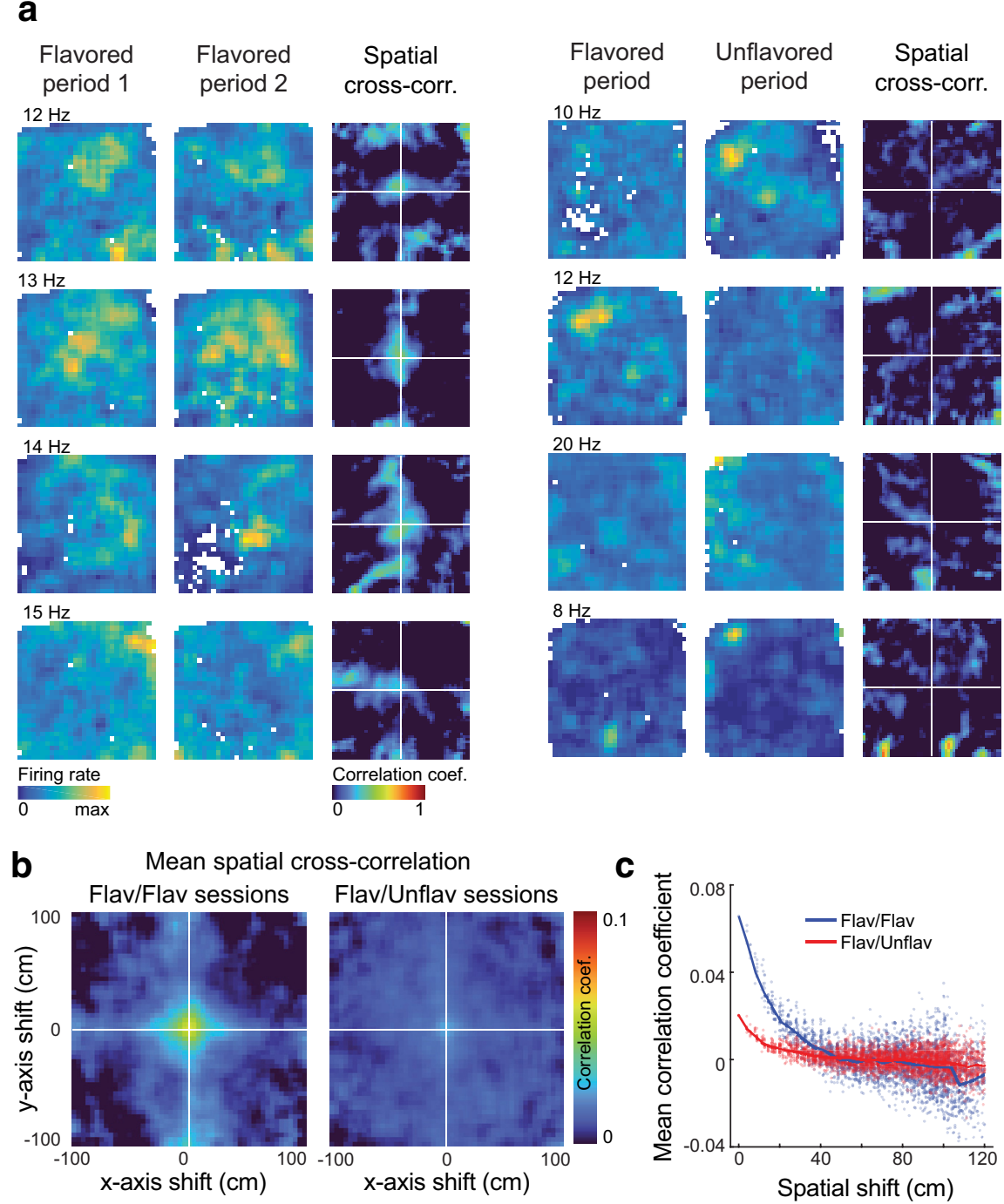

Figure 5. Firing rate map cross-correlations. $\boldsymbol{a}$, Firing rate maps and cross-correlograms are plotted for four neurons recorded in flavored-flavored sessions (left) and four neurons recorded during flavored-unflavored sessions (right). The maximum firing rate across both session periods is plotted near the upper left corner of the firing rate map. Neurons recorded during flavored-flavored sessions tend to show more similar representations across foraging periods, resulting in high correla-

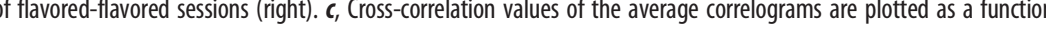
sion type. Flav, Flavored; Unflav, unflavored; cross-corr., cross-correlation.

during the flavored portion, while $6 \%$ of cells ( 18 of 302 cells; $p=0.43$, two-tailed binomial test) were significant in the unflavored session periods (Fig. 7a). None of these proportions differed significantly from what would be expected by chance, suggesting that, although some examples could be identified in this task (Fig. $7 b$ ), grid-like representations were an uncommon motif in the representations of the OFC neurons sampled by our recording electrodes.

\section{Comparison to hippocampal spatial coding}

Last, it is interesting to consider how spatial coding in OFC compares with the canonical spatial representations found in hippocampal neurons. To facilitate this comparison, we performed the same analyses described above on a freely available dataset of 148 
a
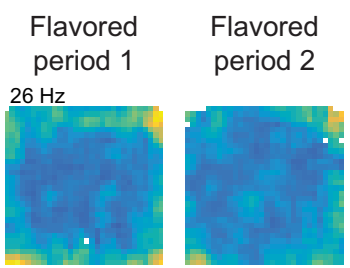

Temporal
correlation
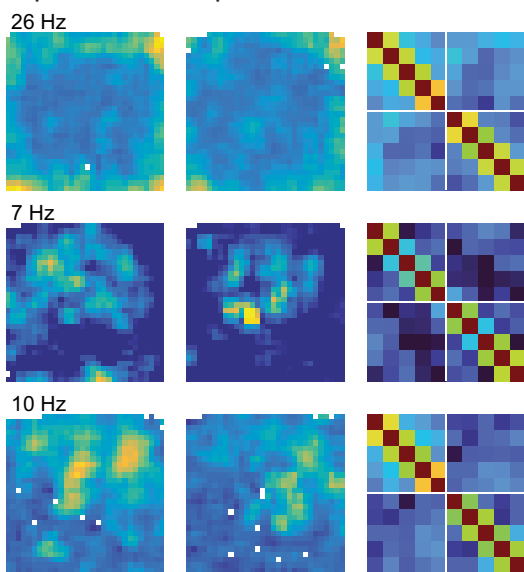

$15 \mathrm{~Hz}$

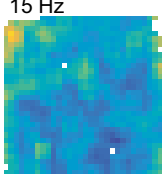

Firing rate

0 max

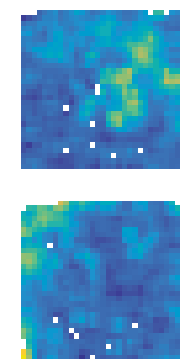

b

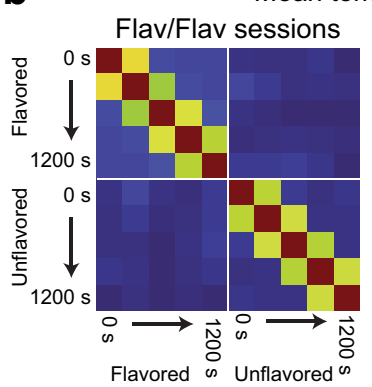

Mean temporal correlation
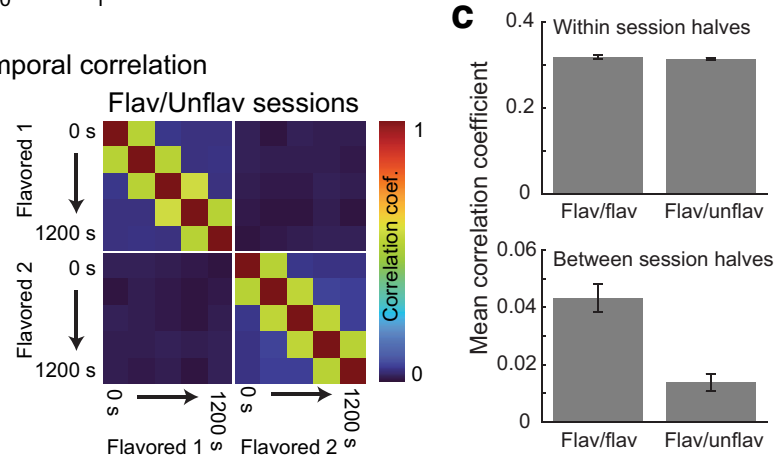

Figure 6. Temporal correlations. $\boldsymbol{a}$, Firing rate maps and correlograms are plotted for four OFC neurons recorded during flavored-flavored sessions (left column) and four neurons recorded during flavored-unflavored sessions (right column). The maximum firing rate across both session periods is plotted near the upper left corner of the firing rate map. Correlograms were constructed by computing firing rate maps in sliding windows (window size $=6 \mathrm{~min}$, step size $=3.5 \mathrm{~min}$ ) and taking the correlation between all pairs of rate maps. $\boldsymbol{b}$, Mean correlograms were constructed by averaging over all OFC neurons recorded during flavored-flavored sessions and flavored-unflavored sessions. c, Within-context correlations were similarly strong for cells recorded in flavored-flavored and flavored-unflavored sessions. The between-context correlation was significantly stronger for neurons recorded during flavored-flavored sessions. Error bars indicate the SEM. Flav, Flavored; Unflav, unflavored.

dorsal CA1 hippocampal neurons (Mizuseki et al., 2009a,b). These neurons were recorded in rats performing a foraging task for water in an enclosure with the same shape and similar dimensions as ours and therefore provided a reasonable comparison. These hippocampal recording experiments did not include flavor manipulations, so it was not possible to compare representations across flavor contexts; however, we did divide hippocampal recording sessions into two 20 min periods, creating a situation analogous to flavored-flavored sessions in our experiment, where context is held constant over time. It should be noted that subtle differences in experimental procedures and behavioral task demands can have a strong effect on neural activity, so these comparisons, while informative, should be interpreted with care.

Analyzing these data using the same methods applied to the OFC data revealed that hippocampal neurons generally showed stronger and more temporally reliable spatial representations than OFC neurons. Spatial information scores (Fig. 8a,b) were significantly higher in the sample of hippocampal neurons than in OFC neurons recorded in flavored-flavored sessions $\left(t_{(251)}=\right.$ 6.63; $p=2.10 \times 10^{-10} ;$ two-sample $t$ test). The average spatial cross-correlation (Fig. $8 c$ ) at shifts $<20 \mathrm{~cm}$ was significantly greater for hippocampal than for OFC neurons $\left(t_{(251)}=5.71 ; p=3.02\right.$ $\times 10^{-8}$; two-sample $t$ test; Fig. $8 d$ ), as was the average temporal correlation (Fig. $8 e)$, both within $\left(t_{(251)}=5.80 ; p=1.99\right.$ $\times 10^{-8}$; two-sample $t$ test) and between $t_{(251)}=7.20 ; p=6.81 \times 10^{-12}$; two-sample $t$ test) session periods (Fig. 8f).

\section{Discussion}

Striking cognitive and functional similarities between the OFC and hippocampus have led to recent proposals that the two regions interact to encode cognitive maps that provide useful scaffolds for planning complex behaviors (Cohen and Eichenbaum, 1993; Wilson et al., 2014; Wikenheiser and Schoenbaum, 2016; Boorman et al., 2021). Cognitive maps have long been exemplified in hippocampal regions by the encoding of spatial and navigation-related information; place cells, grid cells, and other members of the "hippocampal zoo" are concrete evidence of this general mapping function. To date, no such demonstration has been provided in the OFC. Here we directly addressed this gap in the literature by recording OFC neurons from rats engaged in a classic open-field foraging task (Muller et al., 1987). We found that, as in hippocampus, neurons in the OFC exhibit correlates of place in this task, their activity varying with the location of the rat in the arena. Like classic place fields in other foraging tasks, these neurons have place fields that are temporally stable and uniformly distributed across the enclosure. Direct comparisons to similar recordings from hippocampus indicated that the spatial acuity of the OFC representations, while significant, was weaker than that of the very precise representations in CA1 neurons, and there was little evidence of grid cells in the OFC recordings. Additionally, the spatial encoding in OFC was sensitive to changes in the sensory features of food available in the arena; while a direct comparison to hippocampus for this was not possible, the influence of outcome on OFC representations was substantial. Overall these data provide straightforward evidence of place-like representations in OFC neurons in a task that makes the data easily comparable to data from hippocampal studies. The results are consistent with ideas that OFC formats information so that it can be read out as a cognitive map, while 
at the same time providing interesting contrasts with the cognitive map in hippocampus.

Although directional correlates or correlates of reward locations have been described in previous OFC recording experiments, typically these firing patterns have been confounded by associative information or other features of behavioral tasks occurring at those unique locations (Lipton et al., 1999; Feierstein et al., 2006; Roesch et al., 2006; Steiner and Redish, 2012; Stott and Redish, 2014). Results presented here do not suffer from this shortcoming, since recordings were made in a classic open-field foraging task, and stable and distributed spatial encoding was observed. Thus, these data show that OFC neurons can map a feature space in a manner similar to what has been observed in hippocampus. These data establish a modicum of continuity across lateral and medial parts of frontal cortex in terms of neural representations. Location-specific firing patterns -along with other processes associated with the hippocampus, such theta phase precession (Jones and Wilson, 2005), theta sequences (Tang et al., 2021), and replay of both spatial (Euston et al., 2007; Shin et al., 2019; Kaefer et al., 2020) and nonspatial (Peyrache et al., 2009) factors-are well established in anterior cingulate and medial prefrontal regions of the rat brain. Our results raise the possibility that spatial tuning may be a common organizing principle for activity in frontal cortex generally. Beyond frontal cortex, this work contributes to growing evidence of spatial representations outside of the hippocampal formation proper (Saleem et al., 2018; Esteves et al., 2021; Flossmann and Rochefort, 2021; Long et al., 2021; Long and Zhang, 2021; Peyrache and Duszkiewicz, 2021).

Our results also show that spatial mapping can emerge in brain regions with vastly different organizational and input/output structure, identifying one more functional similarity between OFC and hippocampus. However, comparisons with hippocampal spatial encoding also revealed intriguing differences. Specifically, the weaker spatial representations and likely higher impact of biologically relevant local sensory information about reward in OFC neurons accord well with the general consensus that processing in the OFC is biased to emphasize informationspatial or otherwise-relevant to the goals of the subject, even at the expense of other information in the environment. It is also important to note that while the behavior of rats on the task suggests that rats did not have strong preferences for any of the flavors used to define flavor zones, we cannot rule out the possibility that rats had weaker preferences among the flavors that our behavioral measures were not sensitive enough to detect. As such, the influence of pellet value on OFC spatial representations is an important question for future work to address.

Interestingly, removing outcome flavor as a variable did not result in reduced spatial encoding, but there was a significant turnover in the neurons encoding that information. This shift in the population representing location in the arena lines up well with proposals that OFC provides a "map layer" that incorporates context and even hidden or latent information, allowing the same location-in real or abstract task space-to be discriminated (Wilson et al., 2012; Schuck et al., 2016; Zhou et al., 2019a). Here, the same location is represented very differently by OFC when various flavored pellets are present versus when only a single pellet flavor is present. It is unclear whether hippocampal place cells would similarly discriminate flavor contexts in this way, though it is interesting to note that taste coding and spatial responses partially overlap in the hippocampus (Herzog et al., $2019,2020)$. It is well established, however, that other sorts of contextual changes-defined by changes to both external sensory

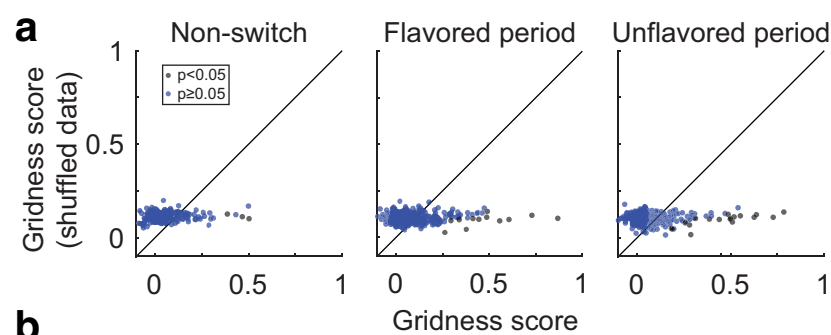

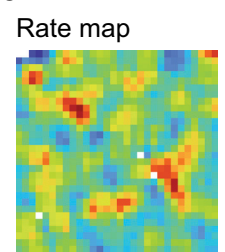

$10 \mathrm{~Hz}$

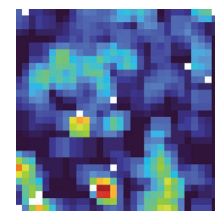

$7 \mathrm{~Hz}$

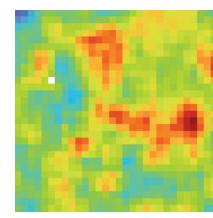

$13 \mathrm{~Hz}$

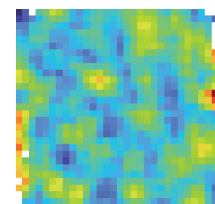

$20 \mathrm{~Hz}$
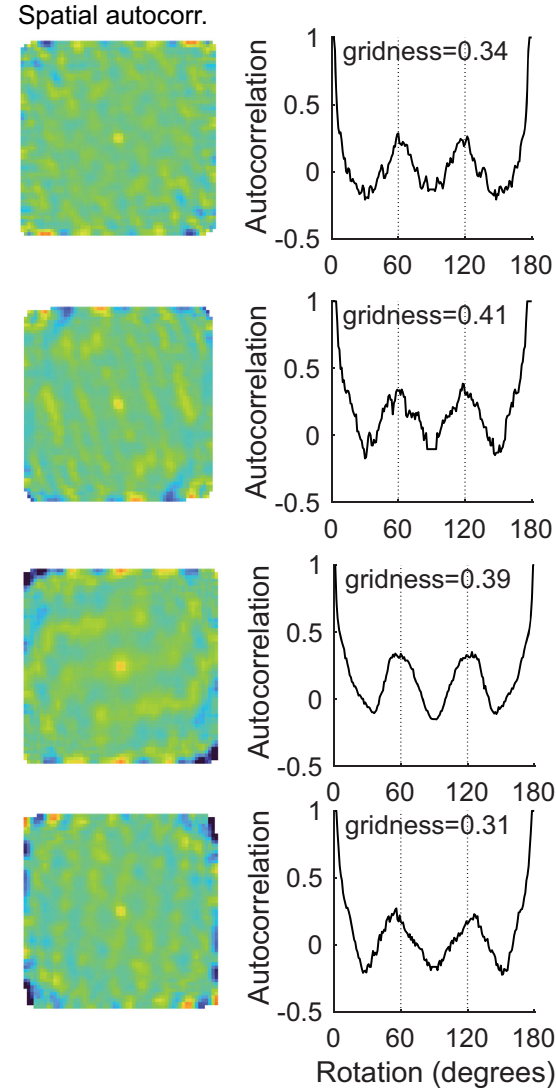

Figure 7. Lack of grid-like representations in OFC neurons. $\boldsymbol{a}$, We computed gridness scores for OFC neurons recorded in nonswitch sessions (left), and separately for flavored (middle) and unflavored (right) portions of flavored-unflavored sessions. Cells were considered to have significant grid tuning if their gridness score exceeded the score computed for $95 \%$ of their randomly shuffled spike trains. Cells that passed this statistical test are plotted with blue dots. Generally, grid tuning was sparse under all behavioral conditions. $\boldsymbol{b}$, Examples of OFC neurons with significant grid tuning are plotted. For each neuron, the firing rate map (left column), and the firing rate map autocorrelation (middle column) were computed. The gridness score was computed based on the strength of correlation between the actual rate map and the rotated rate map (right column). Grid cells are expected to show high correlation values at rotations of $60^{\circ}$ and $120^{\circ}$, and low values at $30^{\circ}, 90^{\circ}$, and $150^{\circ}$. The maximum firing rate of cells is plotted below each rate map. The color scale on autocorrelograms ranges from -0.5 to 0.75 .

cues (Colgin et al., 2008), or internal cognitive (Markus et al., 1995) or motivational (Kennedy and Shapiro, 2009) factorsdrive similar alterations in the spatial representations of hippocampal place cells, a phenomenon termed remapping. In fact, recent work has cast hippocampal remapping as a state inference process (Kubie et al., 2020; Sanders et al., 2020), suggesting another functional similarity hippocampus shares with OFC, which has been characterized as a cognitive map of task state that is especially important for inferring hidden states not fully defined by external sensory inputs (Wilson et al., 2014). 

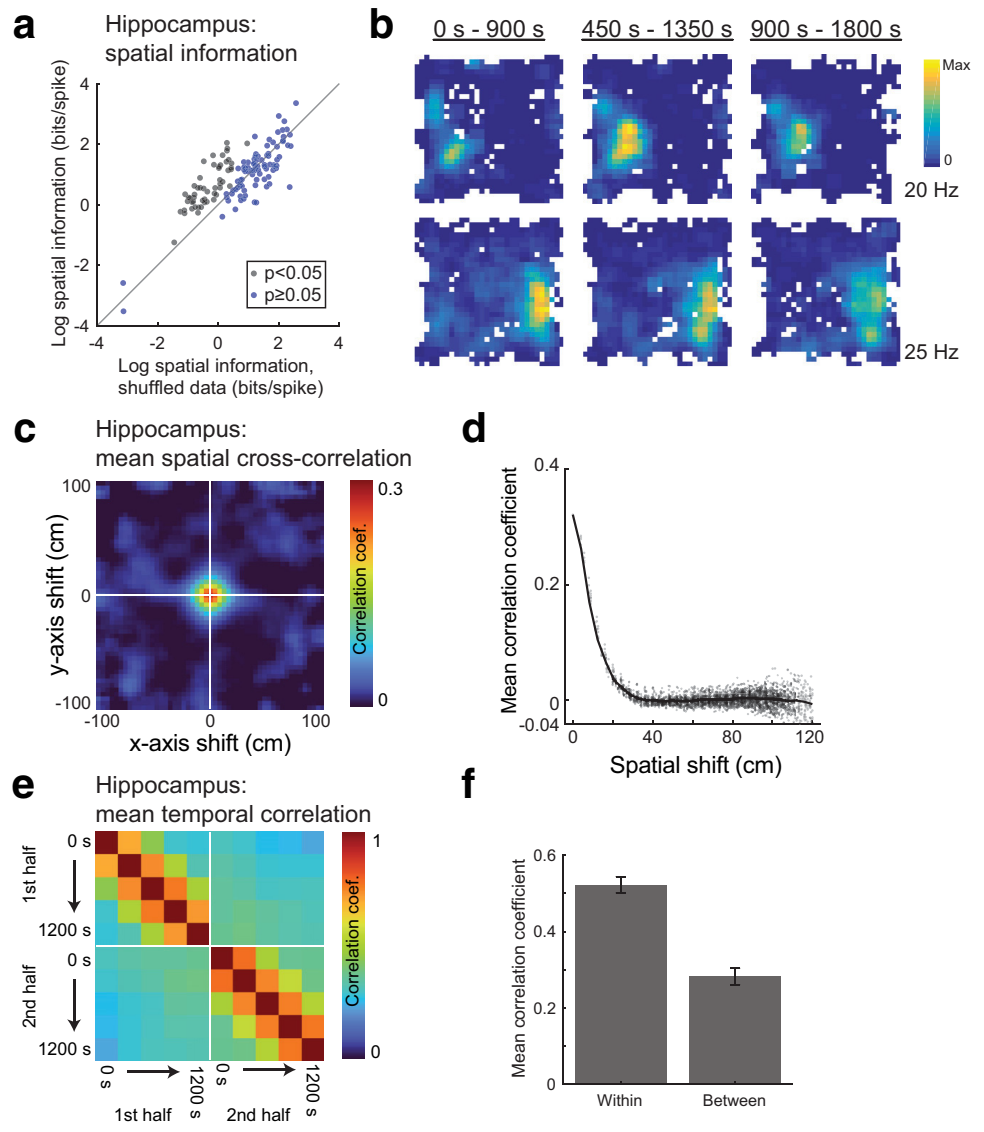

Figure 8. Comparison of hippocampal spatial tuning. $\boldsymbol{a}$, The log spatial information score for each hippocampal neuron is plotted against the mean spatial information score averaged over 500 random shuffles. Neurons plotted with black dots met the statistical criterion for significant spatial information. Compare with OFC data in Figure $3 a$. $\boldsymbol{b}$, Firing rate maps for two hippocampal neurons show stable spatial tuning over a 30 min interval. Compare with OFC examples in Figure $3 b-e$. c, The mean cross-correlogram of hippocampal neuron rate maps computed from the first and second portions of recording sessions. $\boldsymbol{d}$, Cross-correlation strength was greatest for small shifts, indicating stable representations across the first and second portions of recording sessions. Compare with OFC data in Figure $5, a$ and $b$. $\boldsymbol{e}$, Firing rate maps were computed in sliding time bins for hippocampal neurons (as for OFC data in Fig. 6), and the correlation was computed for all pairs of rate maps. Note the high correlation values both along and off the diagonal, indicating stable representations over time. $\boldsymbol{f}$, Average correlation strength was high both for rate maps from the same half of the record ing session ("within context") and for rate maps from different halves of the recording session ("between context").

The absence of significant numbers of grid cells in our dataset is also of interest, given that there are now several reports of gridness in OFC in another measure of neural activity, the BOLD response (Constantinescu et al., 2016; Bao et al., 2019). Although this could reflect species or regional differences between our study and this other work, it might also reflect differences in the basis of the neural measures. One of the useful things about extracellular recording in cortical regions is that fine-wire microelectrodes are biased to pick up activity from large regular spiking neurons likely to be pyramidal output neurons (McCormick et al., 1985; Connors and Gutnick, 1990). BOLD does not appear to suffer from this bias (Logothetis and Wandell, 2004). As a result, one interpretation of the low number of grid cells in our dataset is that the gridness reported in BOLD response reflects the influence of input from hippocampal areas, functioning to help support and properly organize or coordinate the map in OFC with that in other areas (Raithel and Gottfried, 2021). Another possibility is that the foraging task used in the present work was not sufficiently challenging to require or evoke gridlike representations in OFC neurons. In hippocampus, for instance, there is evidence that behavioral demands alter spatial representations, with place cells showing increased temporal reliability on tasks that require solving more challenging navigation problems (Olypher et al., 2002; Jackson and Redish, 2007; Fenton et al., 2010; Wikenheiser and Redish, 2011). Grid-like representations might similarly emerge on behavioral tasks that require decision-making, inference, or generalization (Behrens et al., 2018; Lee et al., 2021).

There is increasing evidence that OFC plays a role in spatial decision-making tasks, and that the hippocampus or hippocampal formation is critical to normal processing in the OFC. Lesions or inactivation of OFC results in behavioral deficits on tasks that include a spatial component (Kolb et al., 1983; Corwin et al., 1994; Young and Shapiro, 2009, 2011). Consolidation of information about task state in human OFC correlates with offline replay of that information in hippocampus (Schuck and Niv, 2019), and OFC-hippocampal interaction is increased during OFC-dependent inference (Wang et al., 2020). Further, when that interaction is disrupted in real time by optogenetic inactivation of hippocampal outflow in subiculum, neurons recorded in rat OFC show a selective reduction in the integration of information about the value, location, and sensory features of expected outcomes was lost (Wikenheiser and Schoenbaum, 2016). The apparent interdependence of OFC and hippocampus for proper representation of task state and the real-time collapse of the normal multidimensional representations in OFC on disruption of that interaction might reflect the loss of the support function provided by the hippocampal formation. The lack of such support leads to behavioral deficits; disconnection of OFC and ventral hippocampal regions causes deficits in reversal learning in rats (Thonnard et al., 2021), and manipulations of OFC targeted to selectively disrupt hippocampal-mediated theta oscillations impair value-based decision-making in monkeys (Knudsen and Wallis, 2020).

Clearly, more work will be necessary to understand the functional importance of spatial representations in OFC. Foremost, more challenging behavioral tasks could provide insight into the cognitive functions these representations support, and causal manipulations of hippocampus and adjacent structures could reveal whether spatial representations in OFC are inherited from spatially tuned neurons in other brain regions or are computed locally. Other processes associated with hippocampal spatial representations, such as remapping, reactivation, coupling of spikes to local field potential oscillations, and more could profitably be investigated in OFC. The extensive literature on hippocampal representations and interactions between hippocampus and other parts of the brain provides a roadmap for such future work and a natural basis for comparison. Answering these and similar questions will define how cognitive maps in hippocampus coordinate with similar, though distinct, representations in OFC to form a convergent, global cognitive map that supports adaptive behavior (Wikenheiser and Schoenbaum, 2016; Boorman et al., 2021; Patai and Spiers, 2021). 


\section{References}

Bao X, Gjorgieva E, Shanahan LK, Howard JD, Kahnt T, Gottfried JA (2019) Grid-like neural representations support olfactory navigation of a twodimensional odor space. Neuron 102:1066-1075.

Barry C, Burgess N (2017) To be a grid cell: shuffling procedures for determining "gridness". BioRxiv 230250.

Behrens TE, Muller TH, Whittington JCR, Mark S, Baram AB, Stachenfeld KL, Kurth-Nelson Z (2018) What is a cognitive map? Organizing knowledge for flexible behavior. Neuron 100:490-509.

Boorman ED, Sweigart SC, Park SA (2021) Cognitive maps and novel inferences: a flexibility hierarchy. Curr Opin Behav Sci 38:141-149.

Bostock E, Muller RU, Kubie JL (1991) Experience-dependent modifications of hippocampal place cell firing. Hippocampus 1:193-205.

Brandon MP, Bogaard AR, Libby CP, Connerney MA, Gupta K, Hasselmo ME (2011) Reduction in theta rhythm dissociates grid cell spatial periodicity from directional tuning. Science 332:595-599.

Cohen NJ, Eichenbaum H (1993) Memory, amnesia, and the hippocampal system. Cambridge, MA: MIT.

Colgin LL, Moser EI, Moser MB (2008) Understanding memory through hippocampal remapping. Trends Neurosci 31:469-477.

Connors BW, Gutnick MJ (1990) Intrinsic firing patterns of diverse neocortical neurons. Trends Neurosci 13:99-104.

Constantinescu AO, O’Reilly JX, Behrens TE (2016) Organizing conceptual knowledge in humans with a gridlike code. Science 352:1464-1468.

Corwin JV, Fussinger M, Meyer RC, King VR, Reep RL (1994) Bilateral destruction of the ventrolateral orbital cortex produces allocentric but not egocentric spatial deficits in rats. Behav Brain Res 61:79-86.

Costa VD, Averbeck BB (2020) Primate orbitofrontal cortex codes information relevant for managing explore-exploit tradeoffs. J Neurosci 40:25532561.

Diehl GW, Hon OJ, Leutgeb S, Leutgeb GK (2017) Grid and nongrid cells in medial entorhinal cortex represent spatial location and environmental features with complementary coding schemes. Neuron 94:83-92.

Esteves IM, Chang H, Neumann AR, Sun J, Mohajerani MH, McNaughton BL (2021) Spatial information encoding across multiple neocortical regions depends on an intact hippocampus. J Neurosci 41:307-319.

Euston DR, Tatsuno M, McNaughton BL (2007) Fast-forward playback of recent memory sequences in prefrontal cortex during sleep. Science 318:1147-1150.

Farovik A, Place RJ, McKenzie S, Porter B, Munro CE, Eichenbaum H (2015) Orbitofrontal cortex encodes memories within value-based schemas and represents contexts that guide memory retrieval. J Neurosci 35:83338344.

Feierstein CE, Quirk MC, Uchida N, Sosulski DL, Mainen ZF (2006) Representation of spatial goals in rat orbitofrontal cortex. Neuron 51:495-507.

Fenton AA, Lytton WW, Barry JM, Lenck-Santini P-P, Zinyuk LE, Kubík S, Bures J, Poucet B, Muller RU, Olypher AV (2010) Attention-like modulation of hippocampus place cell discharge. J Neurosci 30:4613-4625.

Flossmann T, Rochefort NL (2021) Spatial navigation signals in rodent visual cortex. Curr Opin Neurobiol 67:163-173.

Grieves RM, Jeffery KJ (2017) The representation of space in the brain. Behav Processes 135:113-131.

Guise KG, Shapiro ML (2017) Medial prefrontal cortex reduces memory interference by modifying hippocampal encoding. Neuron 94:183-192.

Hafting T, Fyhn M, Molden S, Moser MB, Moser EI (2005) Microstructure of a spatial map in the entorhinal cortex. Nature 436:801-806.

Hasz BM, Redish AD (2020) Spatial encoding in dorsomedial prefrontal cortex and hippocampus is related during deliberation. Hippocampus 30:1194-1208

Herzog LE, Pascual LM, Scott SJ, Mathieson ER, Katz DB, Jadhav SP (2019) Interaction of taste and place coding in the hippocampus. J Neurosci 39:3057-3069.

Herzog LE, Katz DB, Jadhav SP (2020) Refinement and reactivation of a taste-responsive hippocampal network. Curr Biol 30:1306-1311.

Hok V, Save E, Lenck-Santini PP, Poucet B (2005) Coding for spatial goals in the prelimbic/infralimbic area of the rat frontal cortex. Proc Natl Acad Sci U S A 102:4602-4607.

Jackson J, Redish AD (2007) Network dynamics of hippocampal cell-assemblies resemble multiple spatial maps within single tasks. Hippocampus $17: 1209-1229$.
Jones MW, Wilson MA (2005) Phase precession of medial prefrontal cortical activity relative to the hippocampal theta rhythm. Hippocampus 15:867873.

Kaefer K, Nardin M, Blahna K, Csicsvari J (2020) Replay of behavioral sequences in the medial prefrontal cortex during rule switching. Neuron 106:154-165.

Kennedy PJ, Shapiro ML (2009) Motivational states activate distinct hippocampal representations to guide goal-directed behaviors. Proc Natl Acad Sci U S A 106:10805-10810.

Knierim J (2015) The hippocampus. Curr Biol 25:R1116-R1121.

Knudsen EB, Wallis JD (2020) Closed-loop theta stimulation in the orbitofrontal cortex prevents reward-based learning. Neuron 106:537-547.

Kolb B, Sutherland RJ, Whishaw IQ (1983) A comparison of the contributions of the frontal and parietal association cortex to spatial localization in rats. Behav Neurosci 97:13-27.

Kubie JL, Levy ER, Fenton AA (2020) Is hippocampal remapping the physiological basis for context? Hippocampus 30:851-864.

Lee S, Linda QY, Lerman C, Kable JW (2021) Subjective value, not a gridlike code, describes neural activity in ventromedial prefrontal cortex during value-based decision-making. Neuroimage 237:118159.

Lipton PA, Alvarez P, Eichenbaum H (1999) Crossmodal associative memory representations in rodent orbitofrontal cortex. Neuron 22:349-359.

Logothetis NK, Wandell BA (2004) Interpreting the BOLD signal. Annu Rev Physiol 66:735-769.

Long X, Zhang SJ (2021) A novel somatosensory spatial navigation system outside the hippocampal formation. Cell Res 31:649-663.

Long X, Deng B, Cai J, Chen ZS, Zhang SJ (2021) Egocentric asymmetric coding in sensory cortical border cells. BioRxiv 2021.03.11.434952.

Markus EJ, Barnes CA, McNaughton BL, Gladden VL, Skaggs WE (1994) Spatial information content and reliability of hippocampal CA1 neurons: effects of visual input. Hippocampus 4:410-421.

Markus EJ, Qin YL, Leonard B, Skaggs WE, McNaughton BL, Barnes CA (1995) Interactions between location and task affect the spatial and directional firing of hippocampal neurons. J Neurosci 15:7079-7094.

Mashhoori A, Hashemnia S, McNaughton BL, Euston DR, Gruber AJ (2018) Rat anterior cingulate cortex recalls features of remote reward locations after disflavoured reinforcements. Elife 7:e29793.

McCormick DA, Connors BW, Lighthall JW, Prince DA (1985) Comparative electrophysiology of pyramidal and sparsely spiny stellate neurons of the neocortex. J Neurophysiol 54:782-806.

Mizuseki K, Sirota A, Pastalkova E, Buzsáki G (2009a) Multi-unit recordings from the rat hippocampus made during open field foraging. CRCNS Data Sharing. doi: 10.6080/K0Z60KZ9.

Mizuseki K, Sirota A, Pastalkova E, Buzsáki G (2009b) Theta oscillations provide temporal windows for local circuit computation in the entorhinalhippocampal loop. Neuron 64:267-280.

Moser EI, Kropff E, Moser MB (2008) Place cells, grid cells, and the brain's spatial representation system. Annu Rev Neurosci 31:69-89.

Muller RU, Kubie JL (1987) The effects of changes in the environment on the spatial firing of hippocampal complex-spike cells. J Neurosci 7:19511968.

Muller RU, Kubie JL, Ranck JB (1987) Spatial firing patterns of hippocampal complex-spike cells in a fixed environment. J Neurosci 7:1935-1950.

O'Keefe J, Nadel L (1978) The hippocampus as a cognitive map. Oxford, UK: Clarendon.

Olypher AV, Lánský P, Fenton AA (2002) Properties of the extra-positional signal in hippocampal place cell discharge derived from the overdispersion in location-specific firing. Neuroscience 111:553-566.

Padoa-Schioppa C, Assad JA (2006) Neurons in orbitofrontal cortex encode economic value. Nature 441:223-226.

Patai EZ, Spiers HJ (2021) The versatile wayfinder: prefrontal contributions to spatial navigation. Trends Cogn Sci 25:520-533.

Peyrache A, Duszkiewicz AJ (2021) A spatial map out of place. Cell Res 31:605-606.

Peyrache A, Khamassi M, Benchenane K, Wiener SI, Battaglia FP (2009) Replay of rule-learning related neural patterns in the prefrontal cortex during sleep. Nat Neurosci 12:919-926.

Powell NJ, Redish AD (2014) Complex neural codes in rat prelimbic cortex are stable across days on a spatial decision task. Front Behav Neurosci 8:120.

Raithel CU, Gottfried JA (2021) What are grid-like responses doing in the orbitofrontal cortex? Behav Neurosci 135:218-225. 
Ramus SJ, Davis JB, Donahue RJ, Discenza CB, Waite AA (2007) Interactions between the orbitofrontal cortex and the hippocampal memory system during the storage of long-term memory. Ann N Y Acad Sci 1121:216-231.

Redish AD (1999) Beyond the cognitive map: from place cells to episodic memory. Cambridge MA: MIT.

Remondes M, Wilson MA (2013) Cingulate-hippocampus coherence and trajectory coding in a sequential choice task. Neuron 80:1277-1289.

Riceberg JS, Shapiro ML (2017) Orbitofrontal cortex signals expected outcomes with predictive codes when stable contingencies promote the integration of reward history. J Neurosci 37:2010-2021.

Roesch MR, Taylor AR, Schoenbaum G (2006) Encoding of time-discounted rewards in orbitofrontal cortex is independent of value representation. Neuron 51:509-520.

Saleem AB, Diamanti EM, Fournier J, Harris KD, Carandini M (2018) Coherent encoding of subjective spatial position in visual cortex and hippocampus. Nature 562:124-127.

Sanders H, Wilson MA, Gershman SJ (2020) Hippocampal remapping as hidden state inference. Elife 9:e51140.

Sargolini F, Fyhn M, Hafting T, McNaughton BL, Witter MP, Moser MB, Moser EI (2006) Conjunctive representation of position, direction, and velocity in entorhinal cortex. Science 312:758-762.

Schuck NW, Niv Y (2019) Sequential replay of nonspatial task states in human hippocampus. Science 364:eaaw5181.

Schuck NW, Cai MB, Wilson RC, Niv Y (2016) Human orbitofrontal cortex represents a cognitive map of state space. Neuron 91:1402-1412.

Shin JD, Tang W, Jadhav SP (2019) Dynamics of awake hippocampal-prefrontal replay for spatial learning and memory-guided decision making. Neuron 104:1110-1125.

Skaggs WE, McNaughton BL, Gothard KM, Markus EJ (1992) An information-theoretic approach to deciphering the hippocampal code. NIPS'92: proceedings of the 5 th international conference on neural information processing systems (Hanson SJ, Cowan JD, Giles CL, eds), pp 1030-1037. San Francisco: Morgan Kaufmann.

Stalnaker TA, Cooch NK, McDannald MA, Tzu-Lan L, Wied H, Schoenbaum G (2014) Orbitofrontal neurons infer the value and identity of predicted outcomes. Nat Commun 5:3926.

Steiner AP, Redish AD (2012) The road not taken: neural correlates of decision making in orbitofrontal cortex. Front Neurosci 6:131.

Stott JJ, Redish AD (2014) A functional difference in information processing between orbitofrontal cortex and ventral striatum during decision-making behaviour. Philos Trans R Soc Lond B Biol Sci 369: 20130472.
Strait CE, Sleezer BJ, Blanchard TC, Azab H, Castagno MD, Hayden BY (2016) Neuronal selectivity for spatial positions of offers and choices in five reward regions. J Neurophysiol 115:1098-1111.

Tang W, Shin JD, Jadhav SP (2021) Multiple time-scales of decision making in the hippocampus and prefrontal cortex. Elife 10:e66227.

Thonnard D, Callaerts-Vegh Z, D'Hooge R (2021) Effects of orbitofrontal cortex and ventral hippocampus disconnection on spatial reversal learning. Neurosci Lett 750:135711.

Wang F, Schoenbaum G, Kahnt T (2020) Interactions between human orbitofrontal cortex and hippocampus support model-based inference. PLoS Biol 18:e3000578.

Whittington JCR, Muller TH, Mark S, Chen G, Barry C, Burgess N, Behrens TEJ (2019) The Tolman-Eichenbaum Machine: unifying space and relational memory through generalisation in the hippocampal formation. BioRxiv 770495

Wikenheiser AM, Redish AD (2011) Changes in reward contingency modulate the trial-to-trial variability of hippocampal place cells. J Neurophysiol 106:589-598.

Wikenheiser AM, Schoenbaum G (2016) Over the river, through the woods: cognitive maps in the hippocampus and orbitofrontal cortex. Nat Rev Neurosci 17:513-523.

Wilson RC, Takahashi YK, Schoenbaum G, Niv Y (2012) Orbitofrontal cortex as a cognitive map of task space: implications for reversal learning and extinction. Soc Neurosci Abstr 38:289.17.

Wilson RC, Takahashi YK, Schoenbaum G, Niv Y (2014) Orbitofrontal cortex as a cognitive map of task space. Neuron 81:267-279.

Yang L, Masmanidis SC (2020) Differential encoding of action selection by orbitofrontal and striatal population dynamics. J Neurophysiol 124:634644

Young JJ, Shapiro ML (2009) Double dissociation and hierarchical organization of strategy switches and reversals in the rat PFC. Behav Neurosci 123:1028-1035

Young JJ, Shapiro ML (2011) Dynamic coding of goal-directed paths by orbital prefrontal cortex. J Neurosci 31:5989-6000.

Zhou J, Gardner MPH, Stalnaker TA, Ramus SJ, Wikenheiser AM, Niv Y, Schoenbaum G (2019a) Rat orbitofrontal activity contains multiplexed but dissociable representations of value and task structure in an odor sequence task. Curr Biol 29:897-907.

Zhou J, Montesinos-Cartagena M, Wikenheiser AM, Gardner MPH, Niv Y, Schoenbaum G (2019b) Complementary task structure representations in hippocampus and orbitofrontal cortex during an odor sequence task. Curr Biol 29:3402-3409.

Zielinski MC, Shin JD, Jadhav SP (2019) Coherent coding of spatial position mediated by theta oscillations in the hippocampus and prefrontal cortex. J Neurosci 39:4550-4565. 